\title{
Labral Index-Controlling Femoral Size to Evaluate the Relationship Between Relative Labral Size and Acetabular Version
}

\author{
Andrew J. Curley, M.D., Arjun Minhas, M.S., Cooper B. Ehlers, M.S., and \\ William F. Postma, M.D.
}

\begin{abstract}
Purpose: To assess the relationship of acetabular version with the length of the anterior and posterior labrum and to introduce a developed metric, the labral index, as a means of controlling for femoral head size when measuring labral length. Methods: One hundred forty-eight patients who underwent hip arthroscopies for femoroacetabular impingement from October 2017 to October 2019 by a single hip surgeon were retrospectively reviewed. Magnetic resonance imaging arthrogram was used to measure central acetabular version (at the center of the femoral head) and cranial acetabular version (at the midpoint between the acetabular roof and center of the femoral head), as well as labral length at the anterior, posterior, and superolateral locations (3, 9, and 12 o'clock, respectively). Labral index was calculated by dividing anterior, posterior, and superolateral labral lengths by the radius of the femoral head. Pearson correlation analysis was used to assess the association between the 2 continuous variables. A priori alpha of 0.05 was established as the cutoff for significance. Results: Forty-three patients (26 female, 17 male) with an average age of $34.2 \pm 11.6$ years were included in analysis. Increased central anteversion was significantly associated with a larger anterior labral length and index $(P=.023, \mathrm{r}=0.343$ and $.005, \mathrm{r}=0.415$, respectively $)$. Cranial anteversion weakly correlated with a significantly increased anterior labral index $(P=.047, \mathrm{r}=0.304)$ but not with larger anterior labral length $(P=.089, \mathrm{r}=0.262)$. No statistically significant association was present for central or cranial version with posterior or superolateral labral measurements. Lateral center edge angle did not correlate with labral size at any location. Conclusions: In patients undergoing hip arthroscopy for symptomatic femoroacetabular impingement, greater acetabular anteversion was significantly associated but weakly correlated with increased anterior labral length, whereas no association was found between posterior labral length and acetabular version. The labral index may be a useful metric to normalize labral length relative to the radius of the femoral head that warrants further investigation for its clinical utility in guiding labral reconstruction versus labral repair. Level of Evidence: Level IV, diagnostic case series.
\end{abstract}

S everal studies have suggested that decreased femoral head coverage, as measured by the lateral center edge angle (LCEA), is associated with

From MedStar Georgetown University Hospital (A.J.C., W.F.P.) and Georgetown University School of Medicine (A.M., C.B.E.), Washington, DC, U.S.A.

The authors report that they have no conflicts of interest in the authorship and publication of this article. Full ICMJE author disclosure forms are available for this article online, as supplementary material.

Received April 28, 2020; accepted January 25, 2021.

Address correspondence to Arjun Minhas, M.S., Georgetown University School of Medicine, 3900 Reservoir Road NW, Washington, DC 20007.

E-mail:minhasarjun@gmail.com or am3619@georgetown.edu

(C) 2021 THE AUTHORS. Published by Elsevier Inc. on behalf of the Arthroscopy Association of North America. This is an open access article under the CC BY-NC-ND license (http://creativecommons.org/licenses/by-nc-nd/4.0/). 2666-061X/20618

https://doi.org/10.1016/j.asmr.2021.01.020 hypertrophy of the superolateral labrum. ${ }^{1-7}$ Identification of a hypertrophic labrum provides information that may guide preoperative planning for the management of labral pathology. As the understanding of 3dimensional hip morphology has evolved with the increased use of computed tomography imaging, the validity of using the LCEA to assess femoral head coverage has been challenged. ${ }^{8}$ Additional metrics such as the Tönnis angle, Sharp's angle, and angle of inclination aim to provide further information regarding hip morphology and acetabular coverage.

Recent evidence suggests that "dysplasia" is a broad term that is insufficient to describe several different variants observed in patients with hip pain. ${ }^{9,10}$ Observed variants include anterolateral, posterolateral, anterosuperior, posterosuperior, and global deficiencies with either global, focal, or absence of cam lesions. ${ }^{9,10}$ Accurate characterization of acetabular morphology is 
imperative to guide surgical correction of dysplastic hips, and the use of the LCEA as an isolated metric may oversimply appropriate characterization of hips with variegated features of dysplasia., ${ }^{9,10}$

Wilkin et al. ${ }^{11}$ proposed a classification system of acetabular dysplasia comprised of 3 main categories of femoral head undercoverage: anterior, posterior, and lateral. While lateral undercoverage has been welldescribed via the LCEA, there has been renewed interest for investigating anterior and posterior femoral head coverage in the native hip. However, there is a paucity of information regarding the hip's adaptive changes, including change in labral size as a result of anterior or posterior femoral head coverage.

Furthermore, the few studies that assessed labral size have recorded this measurement in isolation, without accounting for the relative size of the adjacent anatomy. ${ }^{1-7}$ Anecdotal reasoning would suggest that an incremental increase in labral length would have a more significant impact on a smaller, rather than a larger, femoral head.

There were 2 main purposes of this study: to assess the relationship of acetabular version with the length of the anterior and posterior labrum, and to introduce a developed metric, the labral index, as a means of controlling for femoral head size when measuring labral length. This study hypothesized that increased acetabular anteversion or retroversion would be associated with hypertrophy of the anterior and posterior labrum, respectively.

\section{Methods}

\section{Patient Selection}

Patients who underwent hip arthroscopies performed for femoroacetabular impingement (FAI) by a single hip surgeon (W.F.P.) from October 2017 to October 2019 were retrospectively reviewed. Patients were excluded for concomitant open hip procedures, previous hip surgery, and radiographic or magnetic resonance imaging (MRI) arthrogram images that were unable for review or were poor quality. Anteroposterior (AP) radiographs of the pelvis were excluded if they were rotated or the superior border of the pubic symphysis and tip of the coccyx was not within 1 to $3 \mathrm{~cm} .{ }^{12}$

\section{MRI and Radiographic Evaluation}

Radiographic measurements were obtained on standard AP pelvis radiographs, including LCEA, Tönnis angle, Sharp's angle, radius of femoral head, femoral neck-shaft angle, and presence of ischial spine sign ${ }^{12}$ (Fig 1). MRI arthrograms were obtained with 3-Tesla magnets following intra-articular administration of dilute gadolinium under fluoroscopic guidance. Standard sequences included whole pelvis coronal short tau inversion recovery and coronal $\mathrm{Tl}$ images, as well as axial T2 fat-suppressed (FS), coronal T1 FS, axial oblique proton-density (PD), sagittal $\mathrm{PD}$, sagittal PD FS, coronal PD FS, and axial oblique Tl FS slices of the affected hip. Superolateral labral length (from the osseous-labral junction to the edge of the labrum) was recorded on a coronal MRI through the center of the femoral head. Additional measurements recorded on axial oblique proton density sequences (Fig 2) included central acetabular version (at the center of the femoral head), cranial acetabular version (at the midpoint between the acetabular roof and center of the femoral head), and anterior and posterior acetabular lengths (recorded at the center of the femoral head). All measurements were taken through eUnity Image Viewer (Client Outlook, Inc., South Burlington, VT) on a desktop computer by one senior resident author (A.J.C.). Intraobserver reliability was not assessed for this study.

Labral index, the measurement of labral length relative to femoral size, was calculated by dividing the labral length at each location (anterior, posterior, and superior) divided by the radius of the femoral head. The anterior, posterior, and superolateral labral length were measured at 3, 9, and 12 o'clock respectively. A circle of best fit was drawn over the femoral head on an AP pelvis radiograph to determine its radius (Fig 3).

\section{Statistical Analysis}

Continuous variables included age, labral index, labral length, version, radius of femoral head, alpha angle, LCEA, Tönnis angle, Sharp's angle, and femoral neck-shaft angle. Categorical variables included sex, laterality, and presence of crossover, posterior wall, or ischial spine sign. Continuous variables were summarized as means and standard deviations, and categorical variables were aggregated as frequencies and percentages. Pearson correlation analysis was used to assess the association between two continuous variables and visually displayed with plots. A priori alpha of 0.05 was established as the cutoff for significance, and SAS 9.4 (SAS Institute, Cary, NC) was used to perform the analysis.

\section{Results}

\section{Patient Results}

One hundred forty-eight potential subjects were identified. After exclusion, there were 43 patients with an average age of $34.2 \pm 11.6$ years ranging from 14.7 to 61.9 years (Table 1$)$. There were $26(60.47 \%)$ female and 17 male $(39.53 \%)$ patients, with the left hip affected in $24(55.81 \%)$ patients and right hip in 19 $(44.19 \%)$ patients. The average LCEA was $28.4 \pm 5.5^{\circ}$ (range, 16-39). On MRI arthrogram evaluation, the average central and cranial versions were $16.3 \pm 5.4^{\circ}$ (range, 3-29) and $9.7 \pm 6.2^{\circ}$ (range, -9 to 21 ), 
Fig 1. Anteroposterior radiographs of a left hip demonstrating (A) lateral center edge angle of $33^{\circ}$, femoral neck shaft angle of $126^{\circ}$, and a circle of best fit with a radius (R); and (B) Tönnis angle of $6^{\circ}$, anterior wall length (A), and posterior wall length $(\mathrm{P})$. Coverage index was calculated using the formula: $(\mathrm{A}+\mathrm{P}) / \mathrm{R}$.
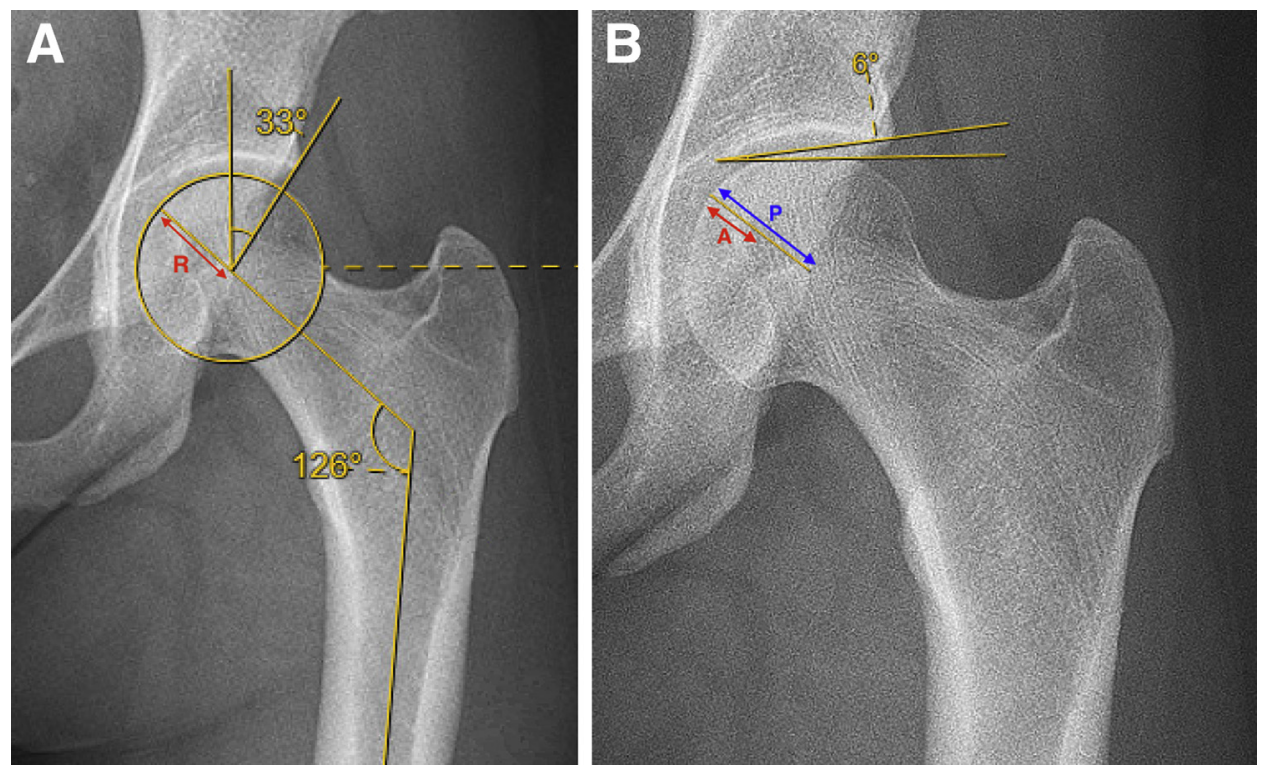

respectively. Mean superolateral labral length was 10.4 $\pm 2.3 \mathrm{~mm}$ (range, 5.8-16.5 mm), mean anterior labrum length was $9.1 \pm 3.0 \mathrm{~mm}$ (range, 2.8-14.7 mm), and mean posterior labrum length was $9.4 \pm 2.2 \mathrm{~mm}$ (range, 5.4-15.3 mm).

\section{Statistical Results}

Increased central anteversion was significantly associated $(P<.05)$ with anterior labral length $(P=.023$, $\mathrm{r}=0.343)$ and anterior labral index $(P=.005, \mathrm{r}=$ 0.415). Cranial anteversion was not significantly correlated with anterior labral length $(P=.09, \mathrm{r}=0.26)$ but was found to be significant for a larger anterior labral index $(P=.047, \mathrm{r}=0.304$, Fig 4$)$. No statistically significant association was present for central or cranial version with posterior or superior labral measurements. No radiographic measurements from this study were significantly associated with labral length at any location.

\section{Discussion}

The most significant finding from this study was that increased acetabular anteversion was associated with greater anterior labral length. This result is consistent with the hypothesis that increased anteversion leads to anterior labral hypertrophy as a compensatory stabilizing mechanism for decreased anterior bony coverage leading to anterior instability. While there are no provided metrics to quantify instability, it is possible that the relationship between acetabular version and labral length was the reason these patients ended up not responding to nonsurgical treatment and undergoing hip arthroscopy, although without a control group it is unknown. In contrast, Garabekyan et al. ${ }^{6}$ did not demonstrate a significant correlation between acetabular version and anterior labral length in a study of 236 symptomatic patients at their hip-preservation clinic. Of note, their study included a larger, more heterogenous population, with nonoperative patients, whereas this
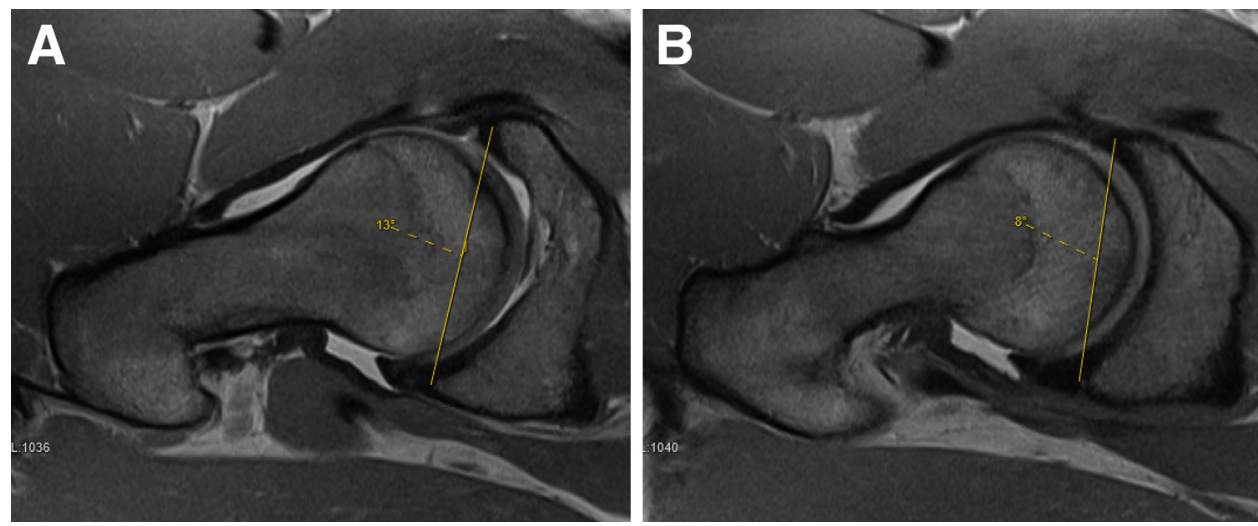

Fig 2. Acetabular version was measured on axial oblique proton density slices through the center of the femoral head for central version (A), as well as the midpoint between the acetabular roof and center of femoral head for cranial version (B). 

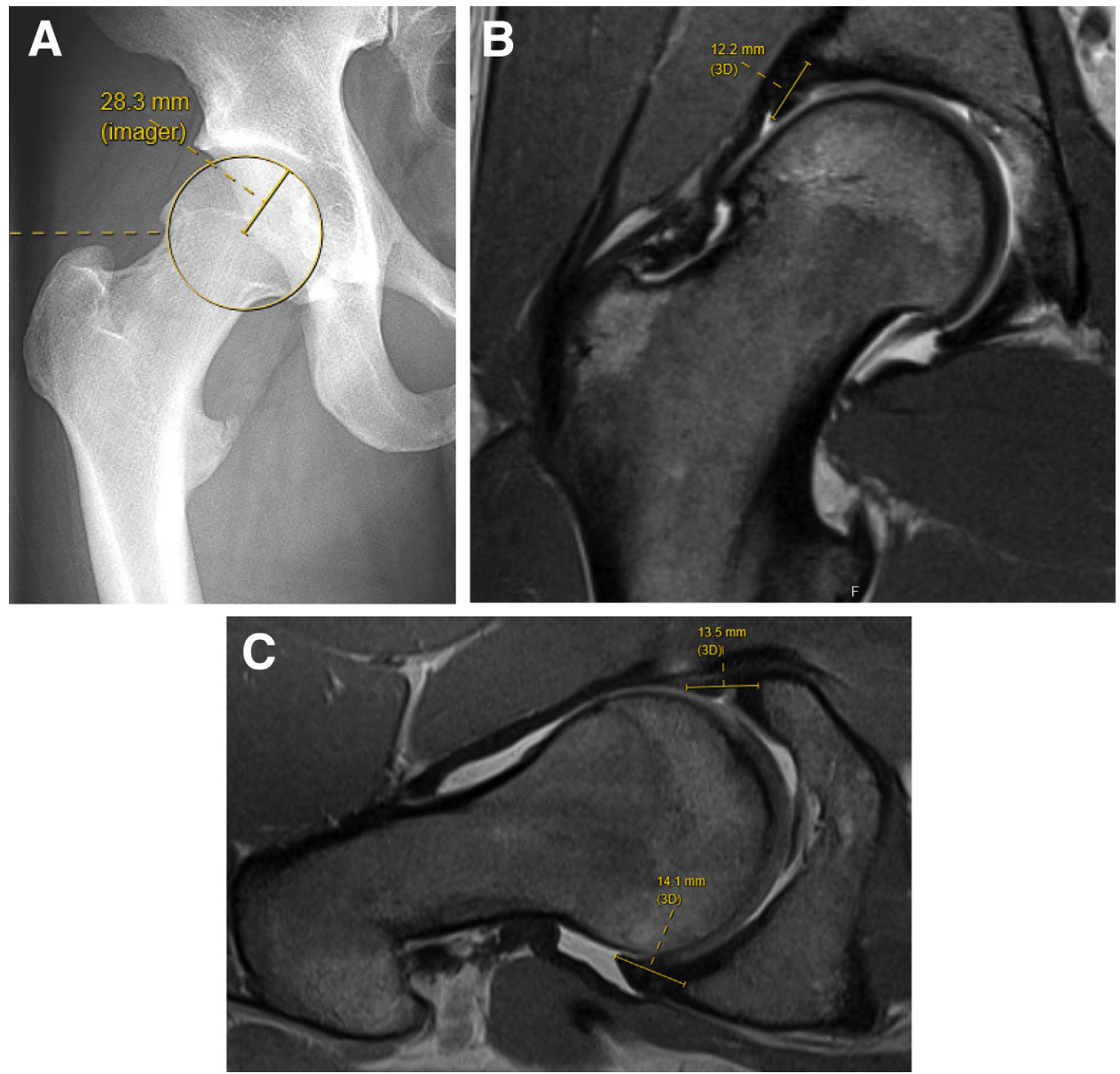

Fig 3. Labral index calculation. Anteroposterior radiograph of the hip demonstrating a circle of best fit with a radius of $28.3 \mathrm{~mm}$ (A). Coronal proton density MRI slice through the center of the femoral head with superolateral labral length (B). Axial oblique proton density MRI slice through the center of the femoral head with anterior and posterior labral lengths (C). Labral Index is calculated by obtaining the labral length at the superolateral (12.2 $\mathrm{mm})$, anterior $(13.5 \mathrm{~mm})$, and posterior $(14.1 \mathrm{~mm})$ locations, then each value is divided by the radius of the femoral head $(28.3 \mathrm{~mm})$ to produce labral indices of $0.43,0.48$, and 0.50 , respectively. (MRI, magnetic resonance imaging.) study was a smaller cohort of patients with FAI symptoms who underwent hip arthroscopy. However, the discrepancy in findings between studies may be attributed to Garabekyan et al. ${ }^{6}$ measuring anterior labral length on axial MRI, whereas this study took this measurement on axial oblique images, as the authors felt it was more in plane with the long axis of the labrum, and it has been shown to have a greater reliability in identification of acetabular labral tears relative to other MRI sequences. ${ }^{13}$ Garabekyan et al. cautioned when examining acetabular version in isolation, as this value is a relative measurement of anterior versus posterior coverage. For example, excessive focal posterior overcoverage or undercoverage (assuming the same anterior coverage) could be falsely interpreted as global acetabular anteversion or retroversion, respectively.

This study aimed to assess the relatively unexplored relationship of posterior labral length with acetabular version. In contrast to the hypothesis, increased posterior labral length was not significantly associated with greater acetabular retroversion. This study is underpowered to truly evaluate retroversion with posterior labral length, as only 10 patients $(23.3 \%)$ had an ischial spine sign consistent with global retroversion. In addition, this study did not assess the combined version of the femur and acetabulum, since the MRI did not include the femoral condyles. It is possible that patients with retroverted acetabulums compensate for posterior instability with greater femoral anteversion, rather than increased posterior labral length.

This study did not observe a significant association between LCEA and labral length at any location. Several studies have found LCEA to be predictive of superolateral labral size. ${ }^{1-7}$ Furthermore, Garabekyan et al. noted that LCEA strongly correlated with labral length on MRI, regardless of location. Similarly, Gupta et $\mathrm{al}^{3}{ }^{3}$ demonstrated that, during intraoperative measurement of labral length in a cohort of 130 hips, LCEA $\leq 25^{\circ}$ was significantly associated with larger labral sizes in all 4 quadrants. A possible explanation for the discrepancy in these results is that this cohort, which only enrolled patients with FAI symptoms, differed from all of the aforementioned studies, which consisted of a broader spectrum of patients, including hips with dysplasia or symptoms of instability.

This study aimed to control for femoral head size when measuring labral length. Interestingly, this study 
Table 1. Demographics and Measurements for the Patients Included in the Analysis $(\mathrm{n}=43)$

\begin{tabular}{lc}
\hline \multicolumn{1}{c}{ Demographics } & \\
\hline Age, y & $34.2 \pm 11.6$ \\
Sex & \\
Female & $26(60.5 \%)$ \\
Male & $17(39.5 \%)$ \\
Laterality & \\
Left & $24(55.8 \%)$ \\
Right & $19(44.2 \%)$ \\
Radiographic measurements & \\
Alpha angle & $62.8 \pm 12.7$ \\
Lateral center edge angle & $28.4 \pm 5.5$ \\
Tönnis angle & $5.9 \pm 4.9$ \\
Sharps angle & $40.2 \pm 3.5$ \\
Femoral neck-shaft angle & $131.1 \pm 5.1$ \\
Radius of femoral head, mm & $25.6 \pm 2.0$ \\
Crossover sign & $27(62.8 \%)$ \\
Posterior wall sign & $13(30.2 \%)$ \\
Ischial spine sign & $10(23.4 \%)$ \\
MRI measurements & \\
Superior labral length, mm & $10.4 \pm 2.3$ \\
Anterior labral length, mm & $9.1 \pm 3.0$ \\
Posterior labral length, mm & $9.4 \pm 2.2$ \\
Superior labral index & $0.41 \pm 0.09$ \\
Anterior labral index & $0.36 \pm 0.12$ \\
Posterior labral index & $0.37 \pm 0.09$ \\
Central version, ${ }^{\circ}$ & $16.3 \pm 5.4$ \\
Cranial version, ${ }^{\circ}$ & $9.7 \pm 6.2$ \\
\hline & \\
\hline &
\end{tabular}

NOTE. All angles and version values are reported in degrees.

MRI, magnetic resonance imaging.

found that greater cranial acetabular anteversion was significantly associated and weakly correlated with an increased anterior labral index $(P=.047, \mathrm{r}=0.304)$ but not a larger anterior labral length $(P=.089, \mathrm{r}=0.262)$. If this study did not use the labral index to normalize the labral length based on the femoral head size, then this relationship would not have been detected. Theoretically, an incremental increase in labral length would provide more coverage on a smaller, rather than a larger, femoral head. For example, when evaluating the smallest $(\mathrm{r}=22 \mathrm{~mm})$ and largest $(\mathrm{r}=30 \mathrm{~mm})$ femoral heads in this cohort, an increase of $5 \mathrm{~mm}$ in labral length would result in $22.7 \%$ versus $16.7 \%$ more coverage, respectively, relative to the radius of the femoral head.

Future work may improve on this study in several ways: elucidation of the relationship of the labral index with symptomatology in patients suffering from FAI, defining a focal anterior or posterior wall coverage abnormality versus a global acetabular version deformity, and investigation of the relationship of combined femoral and acetabular version on the size of the anterior and posterior labrums. Clinically, measurement of the labral index may serve to guide preoperative planning for labral reconstruction versus repair, as it may be a more reliable metric to evaluate the size of the labrum.

\section{Limitations}

A limitation of this study is that it only examined surgical patients with symptoms of FAI. It is possible that the relationship between acetabular version and labral size was the reason these patients failed nonsurgical management, but without a control group this remains unknown. Given that labral hypertrophy is likely a secondary effect of instability, it would be interesting to evaluate anterior and posterior labral length in hips undergoing a redirectional osteotomy for instability, given that these patients may have a stronger correlation between labral length and acetabular version. As only $23.3 \%$ of patients included in this study suffered from global retroversion (evidenced by presence of ischial spine sign), this study is unable to effectively evaluate the relationship of posterior labral length with acetabular version. This study did not calculate McKibbin's index, or evaluate measurements of combined femoral and acetabular version. Theoretically, femoral version can exacerbate or mitigate the impact of excessive acetabular version, which could translate to compensatory changes in labral length. Furthermore, the development of labral hypertrophy is likely a multivariable topic, and this study only examined relationships through bivariate analysis. In addition to the inherent weaknesses of a retrospective design, this study is limited by the lack of patient-reported outcome scores. There was a single senior resident reviewer and intraobserver reliability analysis was not performed for MRI and radiographic evaluation. The clinical significance of anterior labral hypertrophy has yet to be determined, and it is unclear whether these changes correlate with the prevalence of hip symptoms or predict outcomes after surgery.

\section{Conclusions}

In patients undergoing hip arthroscopy for symptomatic FAI, greater acetabular anteversion was significantly associated but weakly correlated with increased anterior labral length, whereas no association was found between posterior labral length and acetabular version. Labral index may be a useful metric to normalize labral length relative to the radius of the femoral head that warrants further investigation for its clinical utility in guiding labral reconstruction versus labral repair.

\section{Acknowledgments}

We thank Haijun Wang, Ph.D., M.S. (Department of Biostatistics and Biomedical Informatics, Medstar 
A

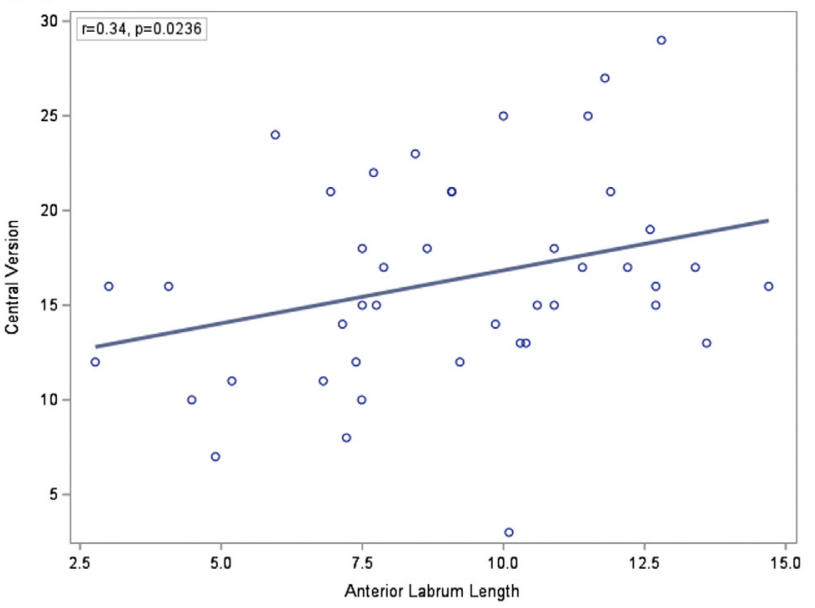

C

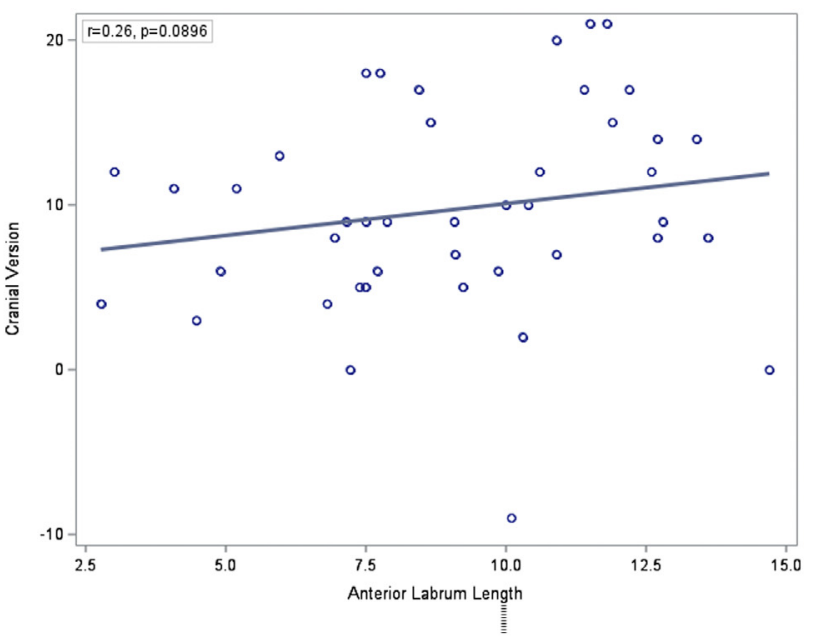

$\mathbf{B}$

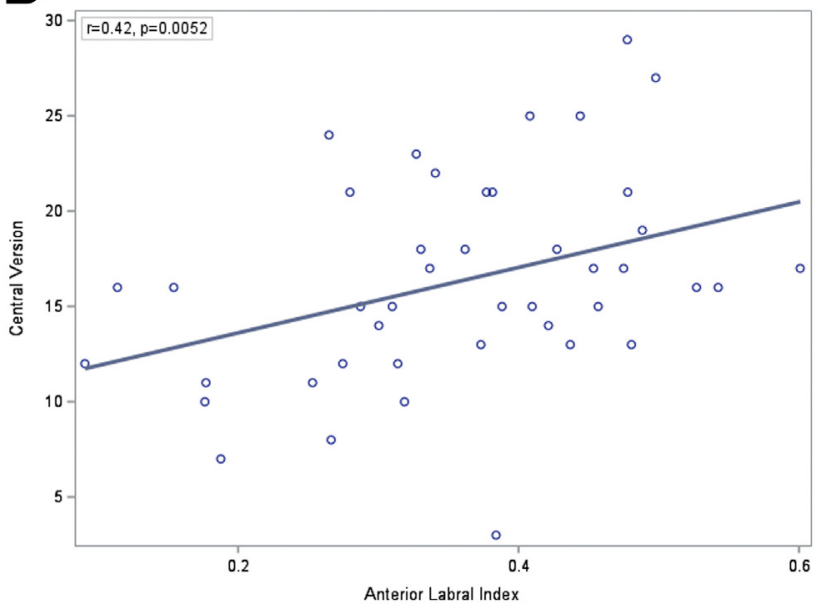

D

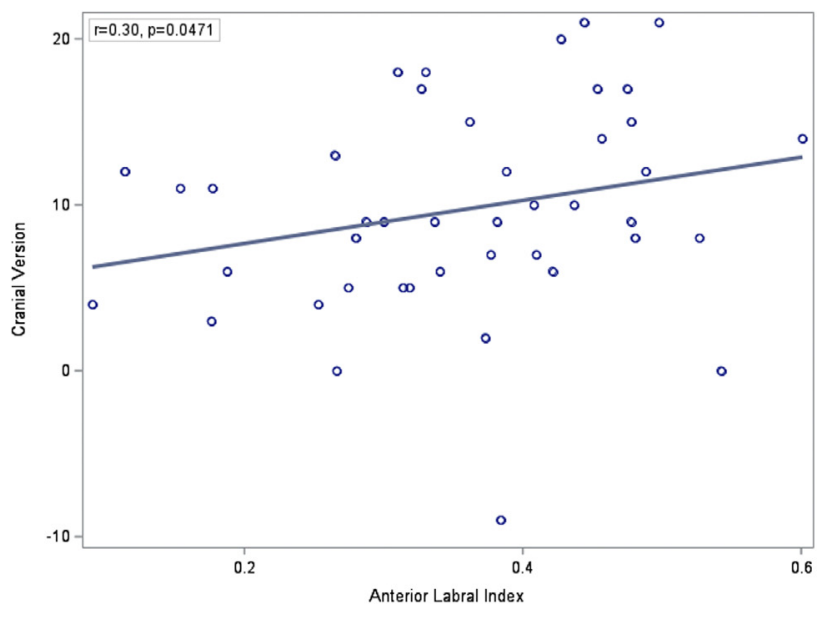

Fig 4. Central version versus anterior labral length $(\mathrm{A})$ and index $(\mathrm{B})$. Cranial version versus anterior labral length $(\mathrm{C})$ and index (D). Pearson correlation analysis was used to assess the association between variables with a priori alpha of 0.05 established as the cutoff for significance. SAS 9.4 (Cary, NC) was used to perform the analysis.

Health Research Institute, Hyattsville, MD) for his support with statistical analysis.

\section{References}

1. Kubo T, Horii M, Yamaguchi J, et al. Acetabular labrum in hip dysplasia evaluated by radial magnetic resonance imaging. J Rheumatol 2000;27:1955-1960.

2. Leunig M, Podeszwa D, Beck M, Werlen S, Ganz R. Magnetic resonance arthrography of labral disorders in hips with dysplasia and impingement. Clin Orthop Relat Res 2004;418:74-80.

3. Gupta A, Chandrasekaran S, Redmond JM, et al. Does labral size correlate with degree of acetabular dysplasia? Orthop J Sports Med 2015;3:2325967115572573.

4. Toft F, Anliker E, Beck M. Is labral hypotrophy correlated with increased acetabular depth? J Hip Preserv Surg 2015;2:175-183.
5. Petersen BD, Wolf B, Lambert JR, et al. Lateral acetabular labral length is inversely related to acetabular coverage as measured by lateral center edge angle of Wiberg. J Hip Preserv Surg 2016;3:190-196.

6. Garabekyan T, Ashwell Z, Chadayammuri V, et al. Lateral acetabular coverage predicts the size of the hip labrum. Am J Sports Med 2016;44:1582-1589.

7. Kraeutler MJ, Goodrich JA, Ashwell ZR, Garabekyan T, Jesse MK, Mei-Dan O. Combined lateral osseolabral coverage is normal in hips with acetabular dysplasia. Arthroscopy 2019;35:800-806.

8. Fritz B. Editorial commentary: Is the lateral center-edge angle sufficient for the approximation of acetabular coverage? Arthroscopy 2019;35:2346-2348.

9. Nepple JJ, Wells J, Ross JR, Bedi A, Schoenecker PL, Clohisy JC. Three patterns of acetabular deficiency are common in young adult patients with acetabular dysplasia. Clin Orthop Relat Res 2017;475:1037-1044.

10. McClincy MP, Wylie JD, Yen YM, Novais EN. Mild or borderline hip dysplasia: Are we characterizing hips 
with a lateral center-edge angle between 18 degrees and 25 degrees appropriately? Am J Sports Med 2019;47:112-122.

11. Wilkin GP, Ibrahim MM, Smit KM, Beaule PE. A contemporary definition of hip dysplasia and structural instability: Toward a comprehensive classification for acetabular dysplasia. J Arthroplasty 2017;32:S20-S27.
12. Clohisy JC, Carlisle JC, Beaulé PE, et al. A systematic approach to the plain radiographic evaluation of the young adult hip. J Bone Joint Surg Am 2008;90:47-66.

13. Ziegert AJ, Blankenbaker DG, De Smet AA, Keene JS, Shinki K, Fine JP. Comparison of standard hip MR arthrographic imaging planes and sequences for detection of arthroscopically proven labral tear. AJR Am J Roentgenol 2009;192:1397-1400. 